\title{
Propagation of Magnetic Clouds - MHD Simulations of Real Events
}

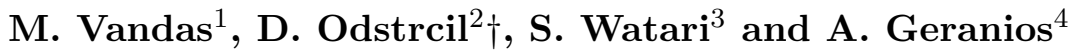 \\ ${ }^{1}$ Astronomical Institute, 14131 Praha 4, Czech Republic \\ email: vandas@ig.cas.cz \\ ${ }^{2}$ University of Colorado/CIRES and NOAA/Space Environment Center, \\ Boulder, CO 80305, USA \\ email: Dusan.Odstrcil@noaa.gov \\ ${ }^{3}$ National Institute of Information and Communications Technology, \\ 4-2-1 Nukuikita, Koganei, Tokyo 184-8795, Japan \\ email: watari@nict.go.jp \\ ${ }^{4}$ Physics Department, University of Athens, \\ Panepistimioupoli-Kouponia, Athens 15771, Greece \\ email: ageran@cc.uoa.gr
}

\begin{abstract}
The paper describes our approach to simulations of real interplanetary events, consisting of four steps: (i) determination of background solar wind, (ii) parameterization of a model flux rope, (iii) launching it into the solar wind, and (iv) calculating its propagation and evolution.
\end{abstract}

Keywords. Sun: coronal mass ejections (CMEs), solar wind, MHD.

\section{Simulation procedure}

Events considered are well-defined ICMEs containing an interplanetary flux rope (magnetic cloud) and with known solar sources. Our simulation programs solve time-dependent MHD equations in three dimensional inner heliosphere with a nearly full angular coverage (with exceptions around the solar poles) and a radial span starting from $0.14 \mathrm{AU}$ (super-critical flow) and including 1 AU. The numerical codes are based on a TVD scheme in spherical coordinates (Odstrcil \& Pizzo 1999) and, under development, on a finite volume method with a Riemann solver using an unstructured grid. Our simulation procedure consists of four steps.

\subsection{Determination of the background solar wind for period of an event using photospheric magnetic field measurements}

A quasi-steady state is determined by program runs, which are driven by conditions in the solar wind at the inner boundary. Two approaches are considered for specifying these conditions. A simple one uses a position of the heliospheric neutral sheet and an angular distance of a given point at the inner boundary to specify the radial velocity (Odstrčil, Dryer \& Smith 1998). The second one is based on relationships between the radial velocity and divergence of the magnetic field (Arge \& Pizzo 2000, Odstrcil 2003); see Figure 1, left part.

$\dagger$ On leave from the Astronomical Institute, Ondřejov Observatory, Czech Republic. 

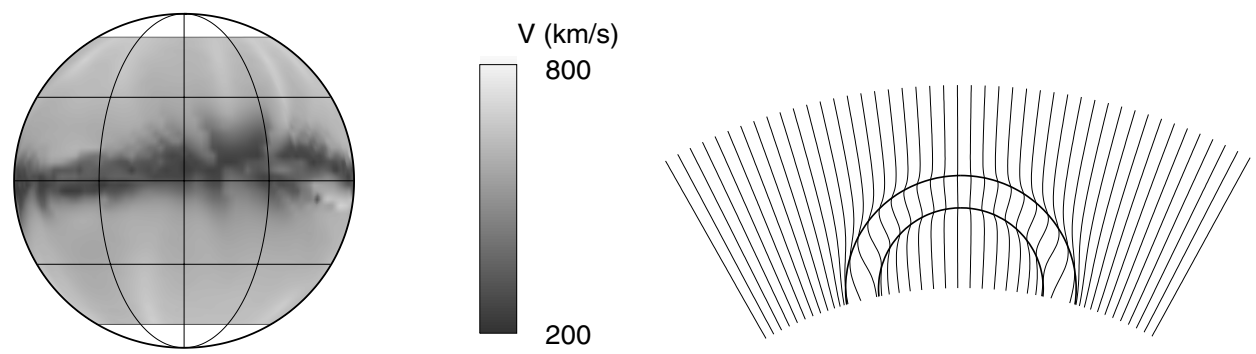

Figure 1. (left) A velocity distribution at the inner boundary based on photospheric magnetic field measurements (the method is described in Arge \& Pizzo 2000 and Odstrcil 2003). (right) A toroidal flux rope merged into a radial field (the method is described in Romashets \& Vandas 2004). Such a configuration can serve for initialization of MHD calculations (the method is described in Vandas, Odstrčil \& Watari 2002).

\subsection{Estimation of flux rope parameters from observations}

It is necessary to determine orientation, size, velocity, magnetic field strength, and chirality of the flux rope. Probably vector magnetographs, coronal field measurements, and STEREO observations may help much in this difficult task.

\subsection{Launching of a model flux rope into the background solar wind}

For a simple field it has been done by Vandas, Odstrčil \& Watari (2002) and by Vandas, Watari \& Geranios (2003). For more realistic fields a method by Romashets \& Vandas (2004) is considered (Figure 1, right part). A flux rope is introduced into the computational domain by time-dependent changes of quantities at the inner boundary.

\subsection{Running numerical simulations and comparing with observations}

Simulations of a flux rope propagation within our scheme in a simple background solar wind (unstructured and unipolar) have been done by Vandas, Odstrčil \& Watari (2002) and by Vandas, Watari \& Geranios (2003). Applications for real events have been and are performed with a model CME without a magnetic structure ("plasma clouds") (Odstrcil, Riley \& Zhao 2004). We are developing procedures to include a magnetic flux rope topology within a more complex solar wind. Several examples of magnetic clouds observed near the Earth and their related CMEs near the Sun, which are well documented in the literature, will be considered in the simulations, the event of May 12, 1997 being the first.

\section{Acknowledgements}

This work is supported by INTAS grant 03-51-6206, AV ČR project S1003006, project ME501 from MŠMT ČR, GA ČR grant 205/03/0953, GA AV ČR grant A3003003, and DOD/AFOSR-MURI project. We also acknowledge support from the bilateral CzechGreek agreement on collaboration in science and technology.

\section{References}

Arge, C. N. \& Pizzo, V. J. 2000, J. Geophys. Res. 105, 10465

Odstrcil, D., Dryer, M. \& Smith, Z. 1998, in: X. S. Feng, F. S. Wei \& M. Dryer (eds.), Advances in Solar Connection with Transient Interplanetary Phenomena, Proceedings of the Third SOLTIP Symposium (Beijing: International Academic Publishers), 191

Odstrcil, D. \& Pizzo, V. J. 1999, J. Geophys. Res. 104, 483

Odstrcil, D. 2003, Adv. Space Res. 32, 497

Odstrcil, D., Riley, P. \& Zhao, X. P. 2004, J. Geophys. Res. 109, A02116 
Romashets, E. P. \& Vandas, M. 2004, this Proceedings

Vandas, M., Odstrčil, D. \& Watari, S. 2002, J. Geophys. Res. 107, A9, SSH 2-1

Vandas, M., Watari, S. \& Geranios, A. 2003, in: M. Velli, R. Bruno \& F. Malara (eds.), Solar Wind Ten, AIP Conf. Proc. 679 (Melville, N. Y.: AIP), 691 